\title{
Automatic Detection and Classification Techniques of Acute Myelogenous Leukemia (AML) Using SVM and Generic Algorithm
}

\author{
V.Nehru, N.D.Bobby, K.S.Rani, Giridhar Reddy, M.Anto Bennet S.
}

\begin{abstract}
Majority of youngsters' having connected online through internet either through computers or by smart phones. After the entry of Jio in the field of internet, the competition began and the cost of internet service became much cheaper andnoweveryone can afford the cost. Latest "Times of India" statistics shows around 59\% of internet users are college students/young men. The trend of going to the physical stores to buy the product is in the decline stage where as the trend of surfing product specification as well as its cost and alternates through online marketing sites is increasing among youngsters. Since, it is more convenient for them to shop anywhere and anytime. Shopping can be done $24 x 7$ and before buying; review of product performance through social media and compare its price through varies alternate sites. There is no compulsion to buy the product while surfingoreven if visited the siteforany number of times. The payment can be made through online transition and products will be delivered to doorstep.Hence shopping through online become a joyful experience and preferred by youngsters.
\end{abstract}

Index Terms: Marketing, Segmentation, Technology and Buying Behaviour.

\section{INTRODUCTION}

Acute Myelogenous Leukemia (AML) is a disease of the blood and bone marrow, the springy tissue inside the bones where platelets are made. It is predominant among grown-ups. As the signs and manifestations of AML are non-explicit in nature and like the indications of other normal issue, it might prompt wrong analysis. Manual minute examination of recolored blood smear or bone marrow suction is the best way to successful conclusion of leukemia. Yet, this technique is tedious and less exact. In the proposed work, a method for programmed recognition and grouping of AML in blood smear is exhibited. K-implies calculation is utilized for division as it is a compelling shading based grouping strategy. Both spatial and ghastly highlights are utilized for arrangement. Hereditary calculation is utilized for streamlining the ghastly highlights. Nearby Ternary Pattern (LTP) is utilized for surface depiction. The proposed framework means to build up a technique to group entire

Revised Manuscript Received on December 22, 2018.

V.Nehru, Assistant professor , Department of CSE, VEL TECH, Chennai-600062

Dr.N.D.Bobby, professor, Department of ECE, Vel Tech High Tech Dr Rangarajan Dr Sakunthala Engineering College, Chennai-600062

K.S.Rani, Assistant Professor, Department of EEE,Vel te Vel Tech High Tech Dr Rangarajan Dr Sakunthala Engineering College, Chennai-600062 Giridhar Reddy, professor, Department of ECE,VEL TECH, Chennai-600062

Dr.M.Anto Bennet, professor, Department of ECE,VEL TECH, Chennai-600062 pictures rather than sub pictures. Highlights of the pictures separated after the utilization of Local Binary Pattern (LBP) and LTP are analyzed. The execution of the classifier is evaluated by testing thirty six pictures.

Keywords: Acute Myelogenous Leukemia (AML), Local Ternary Pattern (LTP), of Local Binary Pattern (LBP), American Society of Hematology (ASH), Support Vector Machine (SVM).

INTRODUCTION

Leukemia is a kind of blood disease that starts in the bone marrow. Bone marrow is the delicate tissue in the focal point of the bones, where platelets are delivered. Develop platelets are called undeveloped cells and impacts. Most platelets develop in the bone marrow and after that move into the veins. Fig1 demonstrates the procedure of development of platelets from immature microorganisms. The bone marrow makes distinctive kinds of platelets. Each sort has a unique capacity: White platelets (leukocytes) are utilized by the body to battle contaminations and other remote substances. Leukocytes are made in the bone marrow. Leukemia prompts an uncontrolled increment in the quantity of white platelets. The malignant cells anticipate solid red cells, platelets, and develop white cells (leukocytes) from being made. Dangerous manifestations would then be able to create as ordinary platelets decrease. The disease cells can spread to the circulatory system and lymph hubs. They can likewise head out to the mind and spinal line (the focal sensory system) and different pieces of the body [1-4].

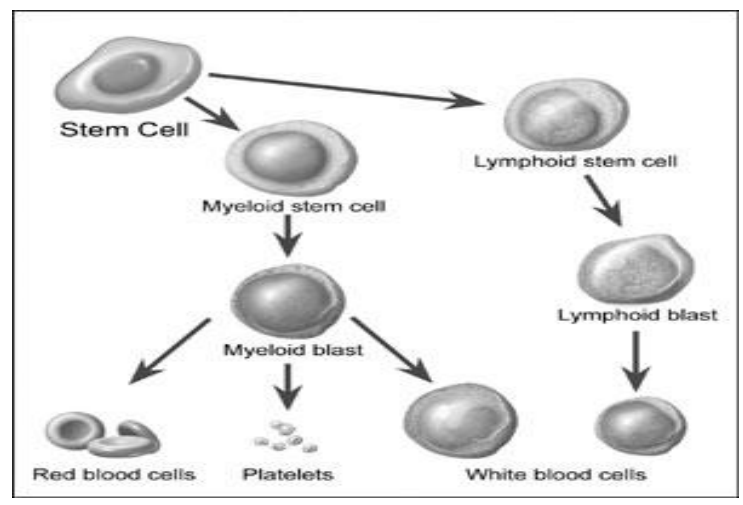

Fig 1.1 Maturation of blood cells

The precise reason for leukemia is obscure. Various types of leukemia are accepted to have diverse causes. Both acquired and natural (non-acquired) factors are accepted to be included. Hazard factors

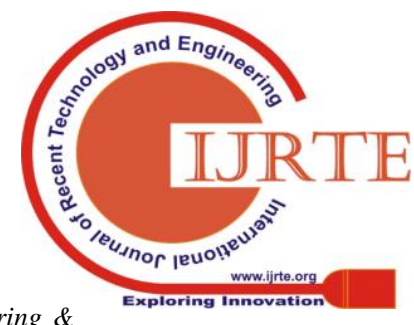

Blue Eyes Intelligence Engineering \& 


\section{Automatic Detection and Classification Techniques of Acute Myelogenous Leukemia (AML) Using SVM and Generic Algorithm}

incorporate smoking, ionizing radiation, a few synthetic concoctions, (for example, benzene), earlier chemotherapy, and Down disorder. Individuals with a family ancestry of leukemia are likewise at higher hazard. Intense is described by a fast increment in the quantity of juvenile platelets. Swarming because of such cells makes the bone marrow unfit to deliver sound platelets. Prompt treatment is required in intense leukemia because of the quick movement and amassing of the threatening cells, which at that point overflow into the circulatory system and spread to different organs of the body. Intense types of leukemia are the most widely recognized types of kids[4-6]. Endless is described by the over the top develop of moderately develop, yet at the same time irregular, white platelets. Regularly taking months or years to advance, the cells are created at an a lot higher rate than typical, bringing about numerous unusual white platelets. Though intense leukemia must be dealt with promptly, interminable structures are some of the time observed for quite a while before treatment to guarantee most extreme viability of treatment. Ceaseless leukemia generally happens in more established individuals, however can hypothetically happen in any age gathering[7-12].

\section{PROPOSED SYSTEM}

The proposed framework gives a strategy for programmed location and grouping of AML. Fig. 2 gives the square outline of the AML classifier. The blood smear pictures are gathered from the online picture bank of American Society of Hematology (ASH). The picture is fragmented utilizing $\mathrm{K}$-implies grouping calculation for isolating the blue cores (locale of enthusiasm) from different cells. Highlights are removed from the divided picture. Both spatial and otherworldly highlights are utilized in the proposed work. Spatial highlights incorporate shape, surface, shading and hausdorff measurement. Shape highlights, surface highlights and hausdorff measurement are removed after the use of LTP administrator on the fragmented picture. Ghostly highlights are acquired by applying wavelet change. Highlights to be utilized for order are chosen after broad PC reenactments. SVM classifier is utilized for order of pictures.

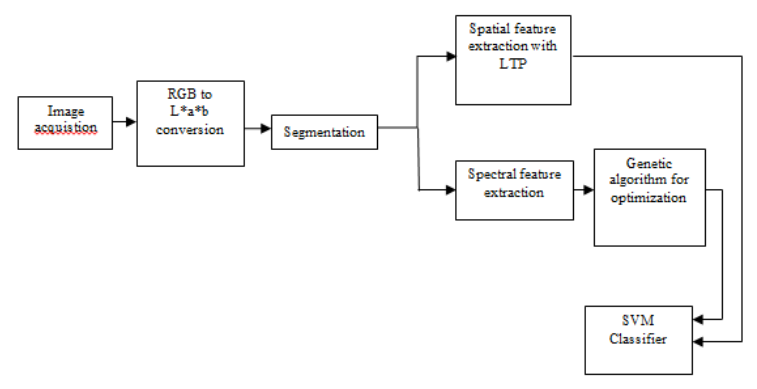

Fig. 2 Block diagram of the AML classifier

The American Society of Hematology is devoted to advancing the getting, analysis, treatment and avoidance of disarranges influencing the blood, bone marrow, the immunologic, haemostatic and vascular frameworks by advancing examination, clinical consideration, instruction and promotion in hematology. The AML pictures utilized in

\section{Image Acquisition}

the work are gotten from the online picture bank of ASH. The ASH picture bank is an electronic picture library that offers thorough and developing accumulations of pictures identifying with a wide scope of hematology classes. They give great pictures caught utilizing diverse magnifying instruments in various goals. The goals utilized in our framework is $184 \times 134$ pixels. Fig.3. (a,b,c,d) gives the delineation of ordinary blood smear and blood smear influenced by AML.

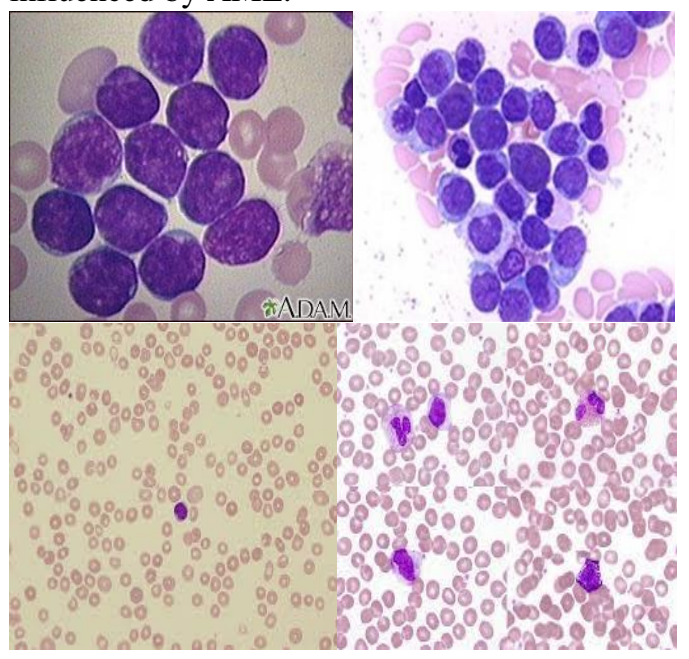

Fig. 3. (a) and (b) Blood smear affected by AML (c) and (d) Normal blood smear

RGB to CIEL*a*b Color Space Conversion:

The pictures created by computerized magnifying lens are as a rule in RGB shading space and are hard to portion. The platelet picture shifts extraordinarily as for shading and force because of numerous reasons, for example, camera settings, differing light, and maturing stain. So as to make the cell division successful and autonomous of these varieties, the RGB input picture is changed over into the CIEL*a*b shading space

\section{SEGMENTATION}

Division is done to extricate essential data from a picture. Prior division calculations for dark dimension pictures have been created. Afterward, it was demonstrated that shading pictures give powerful and dependable division than dim dimension pictures. A portion of the frameworks utilized fluffy based two phase shading division. Yet, it is a semi-managed calculation. Here, K-implies grouping calculation is utilized for division. The proposed framework utilizes three groups comparing to core, foundation and different cells. Each pixel is appointed to one of the groups dependent on the properties of bunch focus. K-implies bunching is a parceling strategy. The calculation allotments information into k totally unrelated groups, and returns the list of the bunch to which it has allocated every perception. Each bunch in the segment is characterized by its part protests and by its centroid, or focus. The centroid for each group is the point to which the total of separations from all articles in that bunch is limited. Every pixel of an item is ordered into $\mathrm{k}$ bunches dependent on the comparing *a and $*$ b values in the $\mathrm{L}^{*} \mathrm{a} * \mathrm{~b}$ shading space. In 
this way, every pixel in the $\mathrm{L}^{*} \mathrm{a} * \mathrm{~b}$ shading space is ordered into any of the $\mathrm{k}$ groups by ascertaining the Euclidean separation between the pixel and each shading marker. These groups relate to core (high immersion), foundation (high luminance and low immersion), and different cells (e. g., erythrocytes and leukocyte cytoplasm). We consider just the bunch that contains the blue core, which is required for the element extraction. While performing k-implies division of complete pictures, it was seen that, in a portion of the fragmented pictures, just the edges of the cores were acquired rather than the entire pictures of the cores. This deficiency was overwhelmed by utilizing morphological separating. Fig 4 (a,b,c,d) gives the info picture, an and b part and divided core.

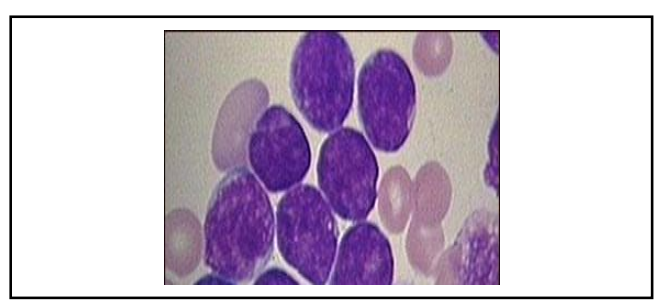

Fig. 4 (a) input image (b) a*component

(c) $b^{*}$ component (d) nucleus

Local Ternary Pattern (LTP):

Nearby Ternary Pattern is a technique utilized for surface order. The work utilizes LBP for surface depiction. LBP can accomplish viable portrayal capacity with appearance invariance and flexibility of example coordinating based strategies. Be that as it may, LBP just edges the differential qualities between neighbourhood pixels and the engaged one to 0 or 1 , which is delicate to commotion existing in the prepared picture. In the proposed work LTP is utilized, which thinks about the differential qualities between neighbourhood pixels and the engaged one as negative or positive improvement if irrefutably the differential esteem is extensive; generally no boost (set as 0). LTPs are an augmentation of LBP. Dissimilar to LBP, it doesn't limit the pixels into 0 and 1 , rather it utilizes an edge consistent to edge pixels into three qualities. Fig. 5 (a,b) demonstrates the fragmented picture after the use of these examples. The shape, shading and surface highlights are extricated after the use of LTP administrator.

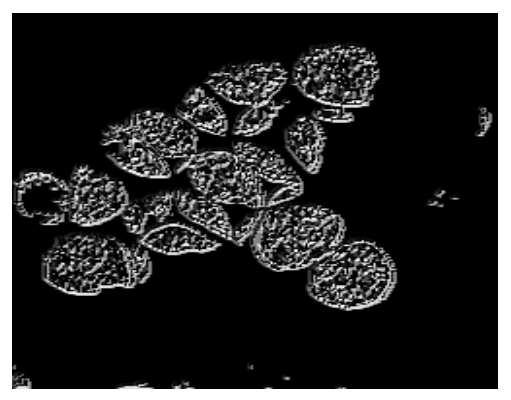

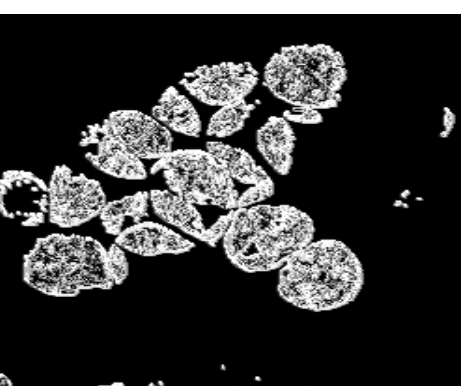

Fig. 5. (a) LTP upper image (b) LTP lower image Genetic Algorithm

Fig. 6 shows the steps involved in a simple genetic algorithm. The brief description of these steps are given below.

The Genetic Algorithm (GA) consists of the following five steps :

Start with a randomly generated population of $\mathrm{N}$ chromosomes, where $\mathrm{N}$ is the size of population

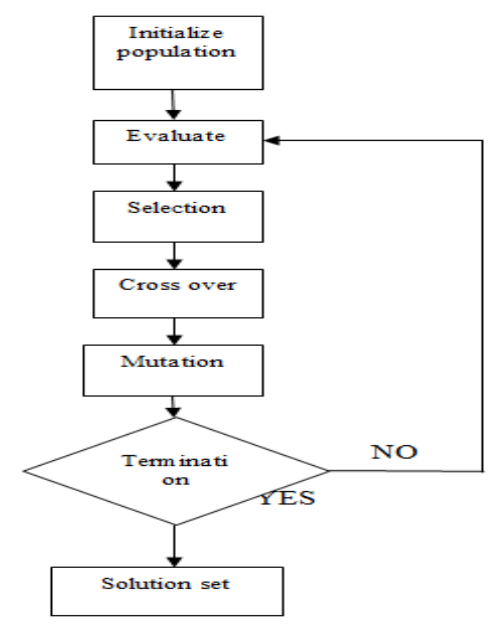

Fig. 6. Steps involved in genetic algorithm

2. Calculate the fitness value of function of each chromosome in the population.

3. Repeat until $\mathrm{N}$ offsprings are created:

3a. Probabilistically select a pair of chromosomes from current population using value of fitness function.

3b. Produce an offspring using crossover and mutation operators.

4. Replace current population with newly created one.

5. Go to step 2.

The approximation and detail coefficients generated are given as the input to genetic algorithm. They are optimized as a set of sixteen coefficients. These features are then used for classification.

\section{SVM CLASSIFICATION}

The selection of classification technique is very crucial as an appropriate choice help improving the accuracy. There is a plenty of statistical techniques, which aim at solving binary classification tasks. In the proposed system, Support Vector Machine (SVM) is used for classification. It constructs a decision surface in the feature space that bisects the two categories, i.e., cancerous and noncancerous, and maximizes

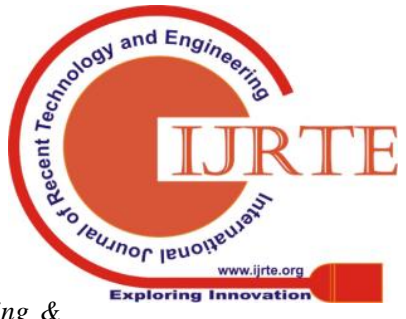




\section{Automatic Detection and Classification Techniques of Acute Myelogenous Leukemia (AML) Using SVM and Generic Algorithm}

the margin of separation between two classes of points . An SVM is primarily a two-class classifier. It can be either linear or non-linear. Here, linear SVM classifier is used as it is computationally inexpensive and it achieves good performance. Initially SVM classifier is trained by using the features extracted from an AML image. These features are loaded as inputs to the algorithm. When an unknown image is given to the SVM classifier, it compares the feature set of the image given during training and that of the unknown image. Based on this comparison, it gives the result of classification. RESULTS OF STEPS INVOLVED IN CLASSIFICATION

The following steps are involved in the classification of AML images:

Step 1: Image Acquisition

The first step in the classification process is the acquisition of image. Images are collected from the online image bank of ASH. The database used in the proposed system consists of 36 images (18 abnormal images and 18 normal images). Fig 7 shows the example of input image.

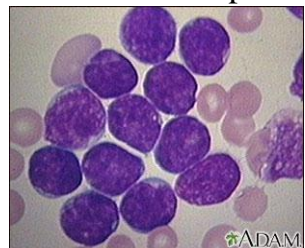

Fig 7. Input image

Step 2: RGB to CIEL*a*b Conversion

The next step is the conversion of the input image in RGB color space to CIEL*a*b color space. Fig $8(a, b)$ shows the images of $\mathrm{a}$ and $\mathrm{b}$ components obtained.

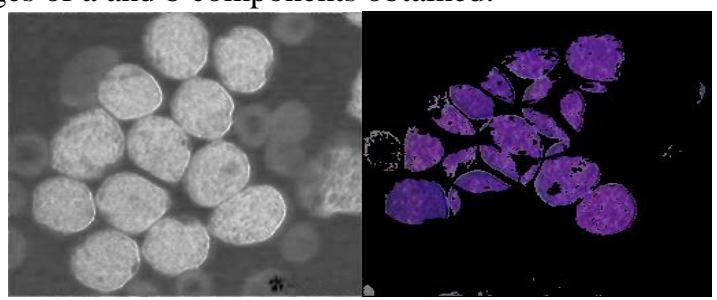

Fig 8 a component b) b component

Step 3: Segmentation

The image is then segmented to separate the blue nucleus from the background and other cells. Fig 9 shows the segmented image with blue nucleus.

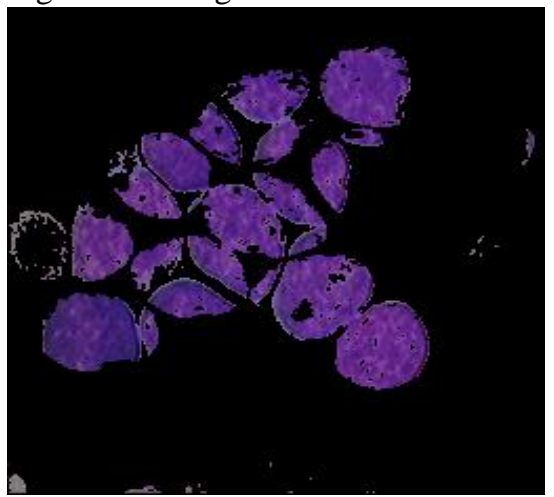

Fig 9 segmented image

Step 4: Feature extraction

The features are extracted from the segmented image. Both spatial and spectral features are extracted.

Spatial features:

Shape, color, texture and hausdorff dimension are the spatial

features obtained. Shape, texture and hausdorff dimension are extracted after the application of LBP and LTP patterns.

LTP and LBP:

LTP and LBP is applied on the segmented image for texture description. Fig $10(a, b)$ shows the segmented image after the application of LTP operator.

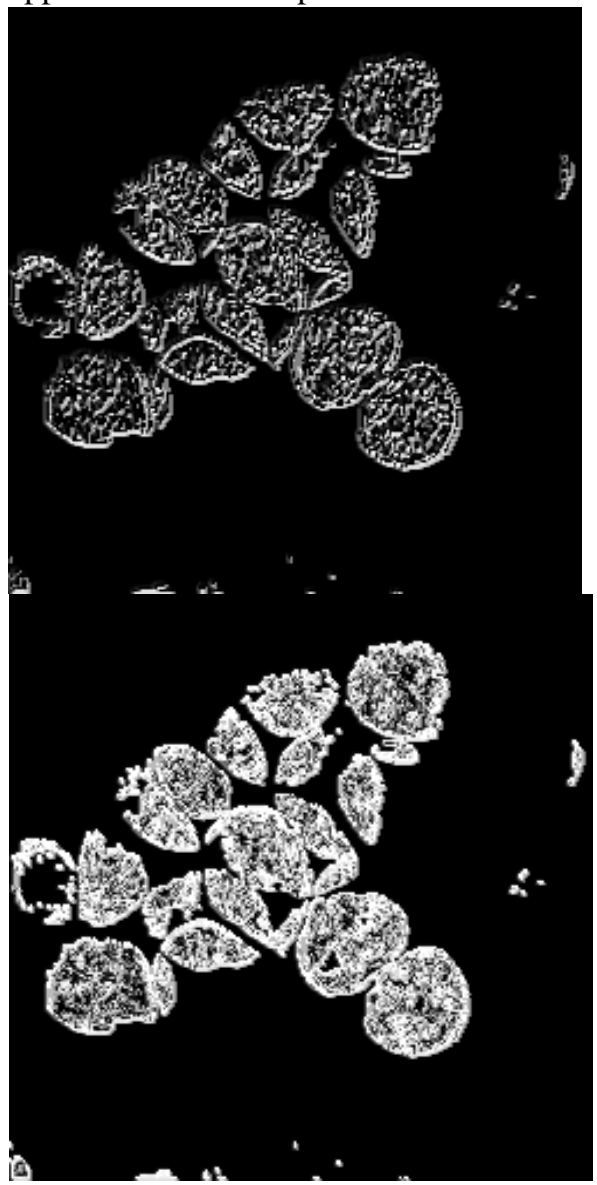

Fig 10 a) LTP upper image b)LTP lower image

Shape features :

Fig 11 shows the plot of shape features obtained from 36 images after the application LBP operator.

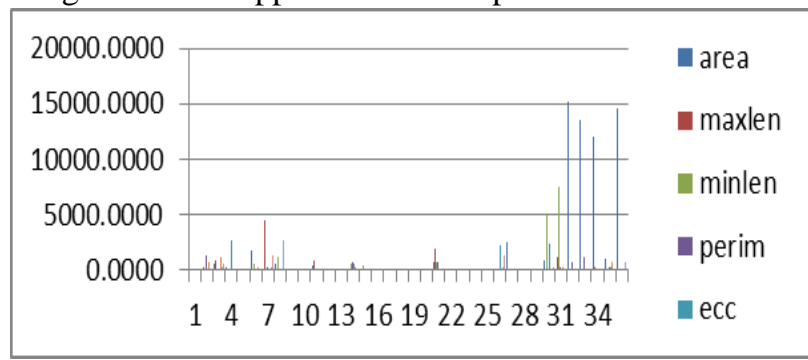

Fig 11 Shape features with LBP pattern

Fig 12 shows the plot of shape features obtained from 36 images after the application if LTP lower pattern.

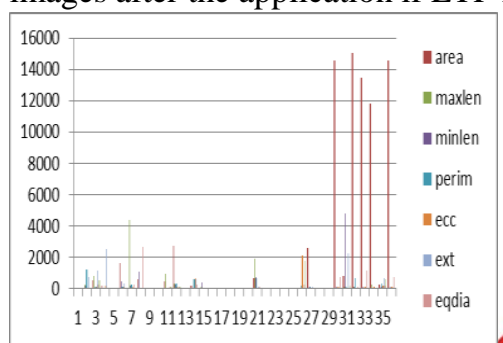

Fig 12 Shape features with LTP lower pattern

Fig 13 shows the plot of 
shape features obtained from the images in the database after the application of LTP upper pattern.

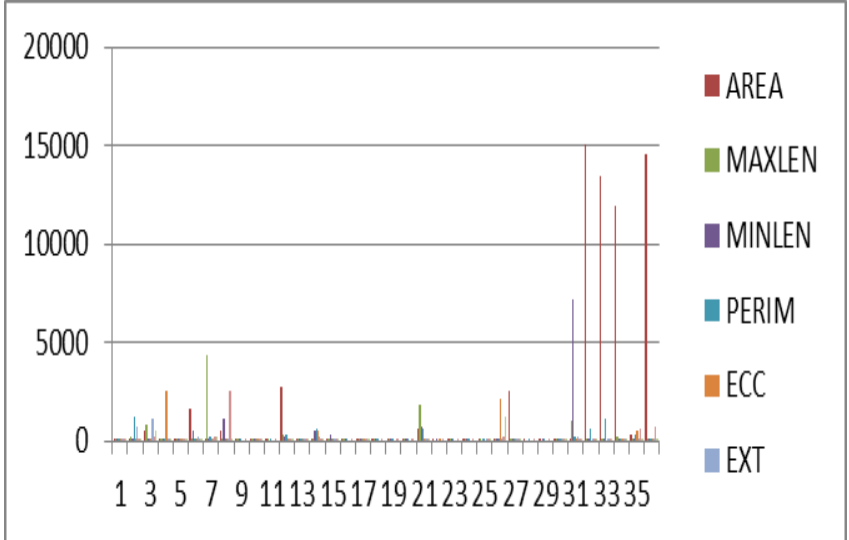

Fig 13 Shape features with LTP upper pattern

From the analysis of the above plots, it can be seen that the shape features obtained after the application of LTP operator differentiates the normal and abnormal images more effectively than the features obtained with LBP pattern. The shape features are extracted from whole images rather than from sub images. So, these features are not more efficient to be used for classification.

Spectral features:

Fig 14 shows the plot of spectral features obtained from the images in the database.

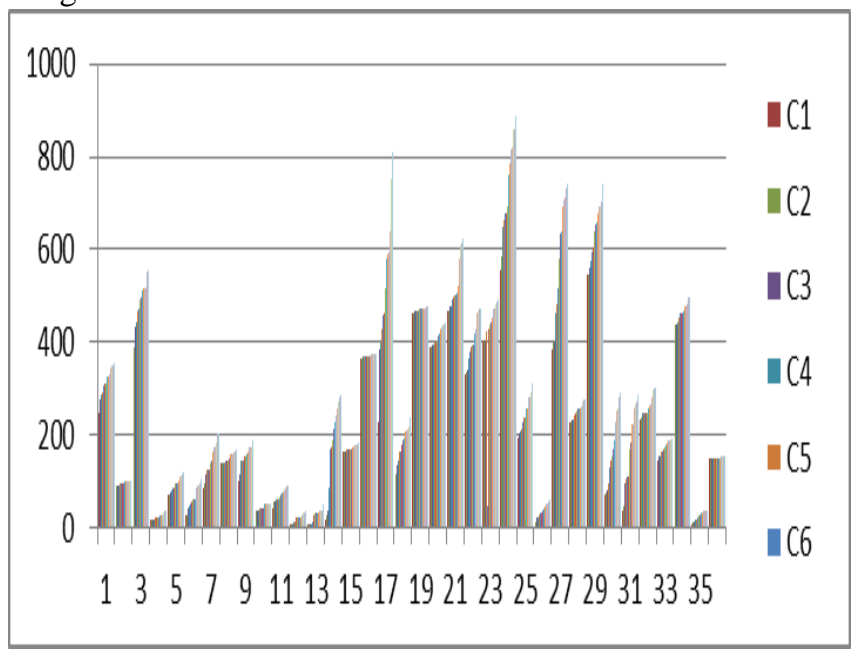

Fig 14 Spectral feature plot

The spectral features are constant and more reliable than spatial features. But the use of approximation co-efficients obtained after applying haar wavelet transform increases the complexity of the classifier. Even though genetic algorithm is applied for optimizing the spectral features, the use of these features affects the confusion matrix of the classifier. In future, this problem can be overcome by using the hyper spectral images and using more efficient wavelets.

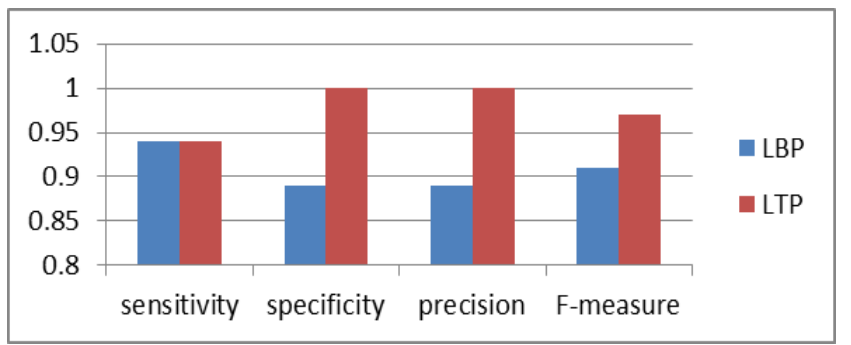

Fig 15 Performance measures of the classifier

From the fig 15, it can be seen that the application of LTP operator has increased the performance of the classifier considerably. Thus, a classifier with improved performance was designed by using LTP for classifying AML images and its performance was analyzed by applying 36 images to the developed system.

\section{CONCLUSION}

The technique for programmed discovery and characterization of AML in blood minute pictures has been introduced. The execution of the technique is assessed by testing 36 pictures of goals $184 \times 138$ pixels got from American Society of Hematology. The proposed framework groups entire blood pictures as opposed to sub pictures. A list of capabilities containing the shape, surface, shading data of the picture is utilized. Also Hausdorff measurement and ghastly highlights were removed. Hereditary calculation was utilized for enhancing otherworldly highlights. Highlights were extricated with the utilization of both LBP and LTP code and the outcomes were looked at. The effect of LTP administrator improved the execution of the classifier significantly. The proposed framework is additionally actualized utilizing FPGA pack. Further research will concentrate on the utilization of phantom highlights as fundamental highlights for order. The total framework will be actualized utilizing equipment. Gathering of more examples to yield better execution will be finished.

\section{REFERENCES}

1. Bruno Direito, Francisco Ventura, Cesar Teixeira and Antonio Dourado, "Optimized feature subsets for epileptic seizure prediction studies," in Proc.Int.Conf.EMBC., pp. 1636-1639, 2011.

2. Chunni Dai and Jingao Liu, "Spectral feature extraction of blood cells based on hyperspectral data," in Proc.Int.Conf.Natural Computation (ICNC)., pp. 1439-1443, 2013.

3. Ilea. D and Whelan. P, "Image segmentation based on the integration of colour-texture descriptors-A review," Pattern Recognit., vol. 44,no. 10/11, pp. 2479-2501, Oct./Nov. 2011.

4. Jing Zhou, Omaru Maruatona. O, and Wei Wang, "Parameter optimization for support vector machine classifier with IO-GA," in Proc. IWCDM, pp. 117-120, 2011.

5. Mohapatra. S and Patra. D, "Automated leukemia detection using hausdorff dimension in blood microscopic images," in Proc.Int.Emerg.Trends Robot Commun.Technol., pp. 64-68, 2010.

6. Dr. AntoBennet, M, Sankar Babu G, Natarajan S, "Reverse Room Techniques for Irreversible Data Hiding”, Journal of Chemical and Pharmaceutical Sciences 08(03): 469-475, September 2015.

7. Dr. AntoBennet, M , Sankaranarayanan S, Sankar Babu G, “ Performance \& Analysis of Effective Iris Recognition System Using Independent Component Analysis", Journal of Chemical and Pharmaceutical Sciences 08(03): 571-576, August 2015.

8. Dr. AntoBennet, M, Suresh R, Mohamed Sulaiman S, "Performance \&analysis of automated removal of head movement artifacts in EEG using brain computer interface", Journal of Chemical and Pharmaceutical Research 07(08): 291-299, August 2015.

9. Dr. AntoBennet, M "A Novel Effective Refined Histogram For Supervised Texure Classification”, International Journal of Computer \& Modern Technology, Issue 01 ,Volume02,pp 67-73, June 2015.

10. Dr. AntoBennet, M, Srinath R, Raisha Banu A, "Development of Deblocking Architectures for block artifact reduction in videos", International Journal of Applied Engineering Research,Volume 10, Number 09 (2015) pp. 6985-6991, April 2015.

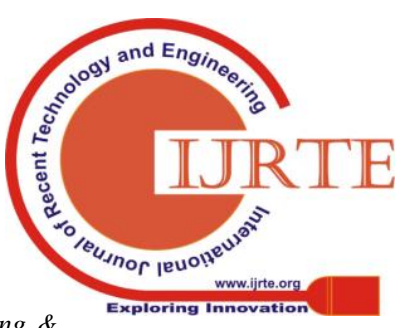

Published By:

Blue Eyes Intelligence Engineering \&

Sciences Publication 
Automatic Detection and Classification Techniques of Acute Myelogenous Leukemia (AML) Using SVM and Generic Algorithm

11. AntoBennet, M \& JacobRaglend, "Performance Analysis Of Filtering Schedule Using Deblocking Filter For The Reduction Of Block Artifacts From MPEQ Compressed Document Images", Journal of Computer Science, vol. 8, no. 9, pp. 1447-1454, 2012.

12. AntoBennet, M \& JacobRaglend, "Performance Analysis of Block Artifact Reduction Scheme Using Pseudo Random Noise Mask Filtering", European Journal of Scientific Research, vol. 66 no.1, pp.120-129, 2011. 